\title{
On the Stabilization of the Wave Equation
}

\author{
Ömer Morgül \\ Bilkent University, Dept. of Electrical and Electronics Engineering \\ 06533, Bilkent, Ankara, Turkey \\ c-mail : morgul@trbilun.bitnet
}

\section{Introduction}

Many mechanical systems, such as spacecraft with flexible attachements, or robots with flexible links, and many practical systems such as power systems, mass transport systems contain certain parts whose dynamic behaviour can be rigorously described only by partial differential equations (PDE). In such systems, to achieve high precision demands, the dynamic effect of the system parts whose behaviour are described by PDE's on the overall system has to be taken into account in designing the controllers.

In recent years, boundary control of systems represented by PDE's has become an important research area. This idea is first applied to the systems represented by the wave equation (e.g. elastic strings, cables), [2], [18], [9], and recently extended to the beam equations, [3], and to the rotating flexiblc structures, [12]. In particular, it has been shown that for a string which is clamped at one cud and is free at the other end, a single non-dynamic boundary control applied at the free end is sufficient to exponentially stabilize the system, [2]. A good source of references to papers in which boundary stabilization problems are treated can be found in [10].

In this note, we consider a linear time invariant system which is represented by one-dimensional wave equation in a bounded domain. We assume that the system is clamped at one end and the bounclary control input is applied at the other end. For this system, we propose a finite dimensional dynamic boundary controller. The transfer function of the controller is restricted to be a strictly positive real function. (We note that this class of controllers has been applied to the stabilization of the Euler-Bernoulli beam equation, see [13]). This class of controllers is quite large and contains some previously proposed non-dynamic controllers as a special case. We show that, if the controller transfer function is strictly proper, the resulting closed-loop system is asymptotically, but not exponentially stable. The main advantage of this type of controller is that the resulting open-loop map is also strictly proper, which is important in proving certain stability robustness results, see [6]. On the other hand, if the controller transfer function is proper but not strictly proper, we show that the resulting closed-loop system is exponcntially stable.

This paper is organized as follows. In the next section we state the stabilization problem. In the section 3, we give the main stabilization results. Then, in the section 4 we present some numerical simulation results, which show the effect of the proposed stabilizing controller on the eigenvalues of the system. Then we give some concluding remarks. 


\section{Problem Statement}

We consider a string as an example of a system whose behaviour is modeled by wave equation. Without loss of generality, we assume that the string length, mass density and the string tension are as $L=1, \rho=1$ and $T=1$, respectively. We denote the displacement of the string by $y(x, t)$ at $x \in(0,1)$ and $t \geq 0$. Futhermore, we assume that the string is fixed at one end and stabilized by dynamic boundary control at the other end. Thus the system under consideration is :

$$
\begin{aligned}
y_{u}(x, t) & =y_{x x}(x, t) \quad x \in(0,1) & t \geq 0 \\
y(0, t)=0 & y_{x}(1, t)=-f(t) & t \geq 0
\end{aligned}
$$

where a subscript, as in $y_{t}$ denotes a partial differential with respect to the corresponding variable, and $f(\cdot): \mathbf{R}_{+} \rightarrow \mathbf{R}$ is the boundary control force applied at the free end of the string. We note that the systems represented by (1), (2) are not restricted to strings; for example vibrations of long cables, the longitudinal motion and the torsional vibrations of elastic beams can also be represented by these equations, see [11]. We assume that $f(t)$ is generated by the following dynamic controller

$$
\begin{array}{rlrl}
\dot{w}(t) & =A w(t)+b y_{l}(1, t) & t \geq 0 \\
f(t)=c^{T} w(t)+d y_{t}(1, t) & t \geq 0
\end{array}
$$

where $w \in \mathbf{R}^{n}$, for some natural number $n$, is the actuator state, $A \in \mathbf{R}^{n \times n}$ is a constant matrix, $b, c \in \mathrm{R}^{n}$ are constant column vectors, $d$ is a constant real number and the superscript $T$ denotes transpose. In case of a non-dynamic actuator, (3) will not exist, and (4) will reduce to

$$
f(t)=d y_{t}(1, t) \quad t \geq 0
$$

which is a case considered by [2]. Therefore, the actuator proposed above may be considered as a generalization of the control law given by (5), see [2], [18], [9].

It is known that if the boundary control force is set to zero (i.e. $f \equiv 0$ ), then the system given by (1)-(2) has infinitely many eigenvalues on the imaginary axis, see e.g. [11]. Our problem is to design an actuator given by (3)-(4) such that the resulting system given by (1)-(4) is stable in some sense.

The stabilization problem stated above can be solved by means of a non-dynamic actuator. For example, in [2], it was proven that the system given by (1)-(2) and (5) is exponentially stable provided that $d>0$. However, it was shown in [5] that the stability of this system is not robust with respect to arbitrarily small delays in the feedback loop. Moreover, the controller transfer function given by (5) is not strictly proper, which might cause some problems in actual implementation, see [6]. While we do not directly address these issues, the above discussion shows that in order to solve a variety of control problems, control laws more general than (5) are required. The main motivation of this paper is to propose a large class of finite dimensional stabilizing controllers for the system given by (1)-(2). The proposed dynamic controller given by (3)-(4) is a candidate for such a class which also covers the controller given by (5).

We also note that the proposed controller offers extra degree of freedom in designing the controllers. This extra degree of freedom could be exploited to solve a variety of control problems, such as 
eigenvalue assignment, disturbance rejection, etc., while maintaining stability, see [17]. Preliminary simulation studies show that by using the controller given by (3)-(4), it could be possible to change the eigenvalues of the system given by (1)-(4) over a specified frequency range, without affecting the rest of the spectrum very much, (see the section 4 ). We note that this could not be achieved by using the controller given by (5), since in this case the spectrum of the system is affected uniformly.

\section{Stability Results}

We first make the following assumptions thoroughout this work :

Assumption 1 : All eigenvalues of $A \in \mathbf{R}^{n \times n}$ have negative real parts.

Assumption $2:(A, b)$ is controllable and $(c, A)$ is observable.

Assumption $3: d \geq 0$; moreover there exists a constant $\gamma, d \geq \gamma \geq 0$, such that the following holds :

$$
d+\operatorname{Re}\left\{c^{T}(j \omega I-A)^{-1} b\right\}>\gamma, \quad, \quad \omega \in \mathbf{R} \square .
$$

If we take the Laplace transform in (4) and (5) and use zero initial conditions, we obtain :

$$
\hat{f}(s)=\left[d+c^{T}(s I-A)^{-1} b\right] \hat{y}_{i}(1, s)=g(s) \hat{y}_{1}(1, s)
$$

where a hat denotes the Laplace transform of the corresponding variable. This, together with (6) implies that the transfer function in (7) is a strictly positive real function, see [19].

Let the assumptions 1-3 stated above hold. Then, it follows from the Meyer-Kalman-Yakubovich Lemma, [19, p.133], that given any symmetric positive definite matrix $Q \in \mathbf{R}^{n \times n}$, there exists a symmetric positive definite matrix $P \in \mathrm{R}^{n \times n}$, a vector $q \in \mathrm{R}^{n}$ and a constant $\epsilon>0$ satisfying :

$$
\begin{gathered}
A^{T} P+P A=-q q^{T}-\epsilon Q \\
P b-c=\sqrt{2(d-\gamma) q}
\end{gathered}
$$

To analyze the system given by (1)-(4), we first define the following function spaces :

$$
\begin{gathered}
\mathcal{H}:=\left\{(u v w)^{\mathbf{T}} \mid u \in \mathbf{H}_{0}^{1}, v \in \mathbf{L}^{2}, w \in \mathbf{R}^{n}\right\} \\
\mathbf{L}^{2}=\left\{f:[0, L] \rightarrow \mathbf{R} \mid \int_{0}^{L} f^{2} d x<\infty\right\} \\
\mathbf{H}_{0}^{k}=\left\{f \in \mathbf{L}^{2} \mid f, f^{\prime}, f^{\prime \prime}, \ldots, f^{(k)} \in \mathbf{L}^{2}, \quad f(0)=0\right\}
\end{gathered}
$$

The equations (1)-(4), can be written in the following abstract form :

$$
\dot{z}=L z \quad, \quad z(0) \in \mathcal{H}
$$

where $z=\left(y y_{t} w\right)^{T} \in \mathcal{H}$, the operator $L: \mathcal{H} \rightarrow \mathcal{H}$ is a linear unbounded operator defined as

$$
L\left(\begin{array}{c}
u \\
v \\
w
\end{array}\right)=\left(\begin{array}{c}
v \\
u_{x x} \\
A w+b v(1)
\end{array}\right)
$$


The domain $D(L)$ of the operator $L$ is defined as :

$$
\begin{aligned}
D(L):= & \left\{(u v w)^{T} \mid u \in \mathbf{H}_{0}^{2}, v \in \mathbf{H}_{0}^{1}, w \in \mathbf{R}^{n} ;\right. \\
& \left.u_{x}(1)+c^{T} w+d v(1)=0\right\}
\end{aligned}
$$

Let the assumptions 1.3 hold, let $Q \in \mathbf{R}^{n \times n}$ be an arbitrary symmetric positive definite matrix and let $P \in \mathrm{R}^{n \times n}, q \in \mathrm{R}^{n}$ be the solutions of (8) and (9) where $P$ is also a symmetric and positive definite matrix. In $\mathcal{H}$, we define the following "energy" inner-product:

$$
\langle z, \bar{z}\rangle_{E}=\frac{1}{2} \int_{0}^{1} y_{t} \tilde{y}_{l} d x+\frac{1}{2} \int_{0}^{1} y_{x} \tilde{y}_{z} d x+\frac{1}{2} \tilde{w}^{T} P w
$$

where $z=\left(y y_{t} w\right)^{T}, \tilde{z}=\left(\tilde{y} \tilde{y}_{t} \tilde{w}\right)^{T}$. We note that $\mathcal{H}$, together with the energy inner-product given by (16) becomes a Hilbert space, [2]. The "energy" norm induced by (16) is :

$$
E(t):=\|z(t)\|_{E}^{2}=\frac{1}{2} \int_{0}^{1} y_{l}^{2} d x+\frac{1}{2} \int_{0}^{1} y_{x}^{2} d x+\frac{1}{2} w^{T} P w
$$

In the sequel we need the following inequality which follows from Jensen's inequality, $[15$, p.110]

$$
y^{2}(x) \leq \int_{0}^{x}\left(y^{\prime}\right)^{2} d s \quad \forall x \in(0,1) \quad \forall y \in \mathbf{H}_{0}^{1}
$$

Lemma 1 : Consider the system given by (1)-(4). Let the assumptions 1-3 hold. Then the energy $E(t)$ given by (17) is a nonincreasing function of time along the classical solutions of (1)-(4). (For the terminology of partial differential equations and semigroup theory, the reader is referred to e.g. [14]).

Proof: By differentiating (17) with respect to time, using (1)-(4), integrating by parts and using (8)-(9), we obtain :

$$
\begin{aligned}
\dot{E} & =\int_{0}^{1} y_{t} y_{t t} d x+\int_{0}^{1} y_{x} y_{x t} d x+\frac{1}{2}\left(\dot{w}^{T} P_{w}+w^{T} P \dot{w}\right) \\
& =-\gamma y_{t}^{2}(1, t)-\frac{1}{2}\left[\sqrt{2(d-\gamma)} y_{t}(1, t)-w^{T} q\right]^{2}-\frac{\rho}{2} w^{T} Q w
\end{aligned}
$$

where to obtain the first equation, we differentiated (17) with respect to time, to obtain the second equation we used (1)-(3), integration by parts, (4),(8), and finally (9).

Since $\dot{E} \leq 0$, it follows that $E(t)$ is a nonincreasing function of time along the classical solutions of (1)-(4). That is, we have $\|z(t)\|_{E} \leq\|z(0)\|_{E}$, hence the system given by (1)-(4) is stable. $\square$

Theorem 1 : Consider the system given by (13), where the operator $L$ is given by (14). Then

i : The operator $L$ generates a $C_{0}$-semigroup $T(t)$ on $\mathcal{H}$,

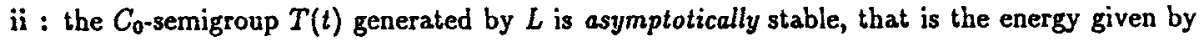
(17) asymptotically tends to zero along the classical solutions of (13).

\section{Proof :}

i : We use Lumer-Phillips theorem, see [14, p.14]. From the Lemma 1 it follows that the operator $L$ is dissipative on $\mathcal{H}$, see (19). Hence, to prove the assertion $\mathrm{i}$, it is enough to show that for some $\lambda>0$, the operator $\lambda I-A: \mathcal{H} \rightarrow \mathcal{H}$ is onto. Let $(f h r)^{\mathrm{T}} \in \mathcal{H}$ be given. We have to find $(u v \boldsymbol{w})^{T} \in D(L)$ such that for some $\lambda>0$ :

$$
\begin{gathered}
\lambda u-v=f \quad, \quad \lambda v-u_{x x}=h \\
\lambda w-A w-b \lambda u(1)=r
\end{gathered}
$$




$$
u(0)=0 \quad u_{x}(1)+c^{T} w+d \lambda u(1)=0
$$

Using (20), we obtain :

$$
\lambda^{2} u-u_{x x}=h+\lambda f
$$

whose solution satisfying $u(0)=0$ is given by :

$$
u(x)=c_{1} \sinh \lambda x-\frac{1}{\lambda} \int_{0}^{x}(h(s)+\lambda f(s)) \sinh \lambda(x-s) d s \quad x \in(0,1)
$$

where $\sinh (\cdot)$ is the hyperbolic sine function. The constant $c_{1}$ can be uniquely determined from (22). The remaining unknowns $v$ and $w$ can be found from (20) and (21), respectively. It can easily be shown that $(u v w)^{T} \in D(L)$. This proves that for all $\lambda>0$, the operator $\lambda I-A: \mathcal{H} \rightarrow \mathcal{H}$ is onto. Since $\mathcal{H}$ is a Hilbert space, it follows that $D(L)$ is dense in $\mathcal{H}$, see [14, p.16]. Hence, by Lumer-Phillips theorem, it follows that $L$ generates a $C_{0}$-semigroup $T(t)$ on $\mathcal{H}$.

ii : To prove the assertion ii, we use LaSalle's invariance principle, extended to infinite dimensional systems, see [16, p.78]. According to this principle, all solutions of (13) asymptotically tend to the maximal invariant subset of the following set :

$$
\mathcal{S}=\{z \in \mathcal{H} \mid \dot{E}=0\}
$$

provided that the solutions are precompact in $\mathcal{H}$. The precompactness of the solutions are guaranteed if the operator $\lambda J-L: \mathcal{H} \rightarrow \mathcal{H}$ is compact for some $\lambda>0$. To prove the last property, we first show that $L^{-1}$ exists and is a compact operator on $\mathcal{H}$. To see this, we put $\lambda=0$ in (20)-(22), which results in the following solution $(u v w)^{T} \in \mathcal{H}$ for any given $(f h r)^{T} \in \mathcal{H} x \in(0,1)$ :

$$
u(x)=-\int_{0}^{x} \int_{0}^{\xi} h(\xi) d \xi d x+c_{2} x \quad, \quad v(x)=-f(x) \quad, \quad w=-A^{-1} r
$$

where the constant $c_{2}$ can be determined from (22). It follows that $L^{-1}$ exists and maps $\mathcal{H}$ into $\mathbf{H}_{0}^{2} \times \mathbf{H}_{0}^{1} \times \mathbf{R}^{n}$, hence is a compact operator. This also proves that the spectrum of $L$ consists entirely of isolated eigenvalues, and that for any $\lambda$ in the resolvent set of $L$, the operator $(\lambda I-L)^{-1}: \mathcal{H} \rightarrow \mathcal{H}$ is a compact operator, see [8, p.187]. Furthermore, our argument above shows that $\lambda=0$ is not an eigenvalue of $L$. This proves that the solutions of (13) are precompact in $\mathcal{H}$, hence by LaSalle's invariance principle, the solutions asymptotically tend to the maximal invariant subset of $\mathcal{S}$ (see (25)).

It can easily be shown that the only classical solution of (13) which lies in $S$ is the zero solution. To see this, we set $\dot{E}=0$ in (19), which results in $w=0$. This implies that $\dot{w}=0$, hence by using (2)-(4) we obtain $y_{t}(1, t)=0, y_{x}(1, t)=0$. Since all boundary conditions are separable, the solution of (1) can be found by using separation of variables. That is, the solution of (1)-(2) with the boundary conditions stated above assumes the form $y(x, t)=A(t) B(x)$ where the functions $A: R_{+} \rightarrow \mathbf{R}$ and $B:[0,1] \rightarrow \mathbf{R}$ are twice differentiable functions to be determined from the boundary conditions. By using (1)-(2) and the boundary conditions stated above, it can easily be shown that either $A \equiv 0$, or $B \equiv 0$, hence $y(x, t) \equiv 0$. Hence, by LaSalle's invariance principle, we conlude that the solutions of (13) asymptotically tend to the zero solution. $\square$

Remark 1 : Let us investigate the system given by (1)-(4) from an input/output point of view. Let us define the boundary control force $f(t)$ as the input and $y_{t}(1, t)$ as the output. If we take the Laplace transform of (1), use zero initial conditions, and use the boundary conditions (2), we obtain 
the plant transfer function $p(s)$ as $p(s)=-\sinh s / \cosh s$. Let $\mathrm{C}$ denote the set of complex numbers, and for $\sigma \in \mathbf{R}$ let $\mathbf{C}_{\sigma^{+}}=\{s \in \mathbf{C} \mid$ Res $\geq \sigma\}$. Although $p(s)$ is not bounded on the imaginary axis, it is bounded on $\mathrm{C}_{\sigma^{+}}$for any $\sigma>0$, hence proper in this sense, $\left(p \in \mathcal{A}_{-}(\infty)\right.$ with the notation of [6], see also [1]). Let us take $d=0$ in the controller given by (4), which results in a strictly proper compansator $g(s)$, see (7). The resulting open-loop map $g(s) p(s)$ is also strictly proper in the sense that for any $\sigma>0, g(s) p(s) \rightarrow 0$ as $|s| \rightarrow \infty, s \in \mathbf{C}_{\sigma^{+}}$, see [6]. Hence, for the system given by (1)-(2), there exist strictly proper controllers for which the open-loop map is also strictly proper and the closed-loop system is stable in asymptotical sense. However, if the stability is understood in exponential sense, following [6], it can be shown that for the system given by (1)-(2) such a controller does not exist. This shows that for infinite dimensional systems, exponential stability requirement may be too restrictive, and that asymptotic stability requirement may be more suitable for designing controllers. This point requires further research.

The above argument shows that when $d=0$, that is the controller given by (7) is strictly proper, the resulting closed-loop system cannot be exponentially stable. In the following we prove that if the controller is proper but not strictly proper, (i.e. $d>0$ ), exponential stability may be obtained. To prove this result, we use the following theorem which is due to Huang, [7] :

Theorem 2 : [4], [7] Let $T(t)$ be a $C_{0}$-semigroup generated by a linear operator $L$ in a Hilbert space. Assume that $T(t)$ satisfies :

$$
\|T(t)\| \leq B \quad t \geq 0
$$

for some $B>0$. Then, there exist $M>0$ and $\delta>0$ such that :

$$
\|T(t)\| \leq M e^{-\delta t} \quad t \geq 0
$$

if and only if the imaginary axis belongs to the resolvent set of $L$, and

$$
\sup _{\omega \in \mathbb{R}}\left\|(j \omega I-L)^{-1}\right\|<\infty \square
$$

For an application of this theorem to flexible structures, see [4].

Theorem 3 : Consider the system given by (13). Assume that in the controller given by (3)-(4) we have $d>0$; moreover there exists a constant $\gamma>0$ such that $d \geq \gamma$ and (6) is satisfied. (cf. with the assumption 3 ). Then the semigroup $T(t)$ generated by the operator $L$ given by (14) is exponentially stable.

Remark 2 : The main distinction between the Theorem 3 and the Theorem 1 is that, in the Theorem 1 , the real part of the transfer function, $\operatorname{Re}(j \omega)$ is required to be strictly positive, whereas in the Theorem 3, it is required to be bounded away from zero. For the non-dynamic case, i.e. when the controller is given by (5) with $d>0$, instead of (3)-(4), the exponential stability was proven in [2]. Hence, the result presented here may be considered as a generalization of that result. However, we note that the techniques we use in the following proof are entirely different than those employed in [2]. We also note that if (4) is replaced by

$$
f(t)=c^{T} w+d y_{i}(1, t)+k y(1, t)
$$

all the results presented in this paper will also hold provided that $k \geq 0$. $\square$ 
Proof : By the Theorem 1, the operator $L$ generates a $C_{0}$-semigroup $T(t)$ on $\mathcal{H}$. By the Lemma 1, this semigroup is bounded, i.e. (27) is satisfied. Also, by the Theorem 1, the spectrum of $L$ consists entirely of countably many isolated eigenvalues, and $\lambda=0$ is not an eigenvalue of $L$.

Next we prove by contradiction that the imaginary axis belongs to the resolvent set of $L$. Suppose that the spectrum of $L$ and the imaginary axis have common points. Since the operator $L$ has point (i.e. discrete) spectrum, it follows that there exists a $\omega \in \mathbf{R}$ such that (20)-(22) has a nontrivial solutions for $\lambda=j \omega$ and $(f h r)^{T}=\left(\begin{array}{lll}0 & 0 & 0\end{array}\right)^{T}$. These solutions are given as :

$$
u(x)=c_{3} \sin \omega x \quad v(x)=j \omega c_{3} \sin \omega x \quad w=c_{3} j \omega \sin \omega(j \omega I-A)^{-1} b \quad x \in(0,1)
$$

where $c_{3}$ is an arbitrary constant. Using (30) in (22) yields :

$$
c_{3}\left[\omega \cos \omega+j \omega\left(c^{T}(j \omega I-A)^{-1} b+d\right) \sin \omega\right]=0
$$

We define $g(j \omega)$ as : $g(j \omega):=R(\omega)+j I(\omega)$ where $R(\omega)$ and $I(\omega)$ are the real and imaginary parts of $g(j \omega)$, respectively (see (7)). Using this in (31), we conclude that either $c_{3}=0$, or the following holds :

$$
\omega \cos \omega-\omega I(\omega) \sin \omega=0 \quad \omega R(\omega) \sin \omega=0
$$

Since $\lambda=0$ is not an eigenvalue of $L$, and since $R(\omega)>\gamma>0, \forall \omega \in \mathbf{R}$, it can easily be shown that (32) does not have any solution. Therefore, in (31) we must have $c_{3}=0$, which, by (30) will yield to the trivial solution. This shows that the imaginary axis does not belong to the spectrum of $L$, hence must belong to the resolvent of $L$, (note that $L$ has point spectrum).

To prove the estimate (29), we first solve (20)-(22) for given $(f h r)^{\boldsymbol{T}} \in \mathcal{H}$ and $\lambda=j \omega, \omega \in \mathbf{R}$. By (24), the solution $u$ which satisfies $u(0)=0$ is given as :

$$
u(x)=j c_{1} \sin \omega x-\frac{1}{\omega} \int_{0}^{x}(h(s)+j \omega f(s)) \sin \omega(x-s) d s \quad x \in(0,1)
$$

where

$$
c_{1}=\frac{\int_{0}^{1}(h(s)+j \omega f(s))(\cos \omega(1-s)+j g(j \omega) \sin \omega(1-s)) d s-c^{T}(j \omega I-A)^{-1} r}{j \omega \cos \omega-w g(j \omega) \sin \omega}
$$

$v(x)$ and $w$ can be found from (20) and (21) as :

$$
\begin{gathered}
v(x)=-\omega c_{1} \sin \omega x-j \int_{0}^{x}(h(s)+j \omega f(s)) \sin \omega(x-s) d s-f(x) \quad x \in(0,1) \\
w=(j \omega I-A)^{-1}[j \omega u(1) b+r]
\end{gathered}
$$

By differentiating (33) with respect to $x$, using integration by parts, and (18), we obtain :

$$
\int_{0}^{2} u_{x}^{2} d x \leq 2 \omega^{2} c_{1}^{2}+K_{1}\left(\int_{0}^{1} h^{2}(s) d s+\int_{0}^{1}\left(f^{\prime}\right)^{2}(s) d s\right)
$$

for some $K_{1}>0$. Similarly, by using integration by parts and (18) in (35), we obtain :

$$
\int_{0}^{1} v^{2} d x \leq 2 \omega^{2} c_{1}^{2}+K_{2}\left(\int_{0}^{1} h^{2}(s) d s+\int_{0}^{1}\left(f^{\prime}\right)^{2}(s) d s\right)
$$

for some $K_{2}>0$. Also, by using (36), (18) and (37), we obtain :

$$
\|w\|^{2} \leq 2\left\|(j \omega I-A)^{-1}\right\|^{2}\left\{\left[2 \omega^{4} c_{1}^{2}+K_{1} \omega^{2}\left(\int_{0}^{1} h^{2}(s) d s+\int_{0}^{1}\left(f^{\prime}\right)^{2}(s) d s\right)\right]\|b\|^{2}+\|r\|^{2}\right\}
$$


To obtain a bound on $\omega c_{1}$ for large $\omega$, we consider the denominator of (34):

$$
D(\omega)^{2}=|j \cos \omega-g(j \omega) \sin \omega|^{2} \geq \gamma^{2} \sin ^{2} \omega+\cos ^{2} \omega-2 I(\omega) \sin \omega \cos \omega
$$

where $|\cdot|$ denotes the absolute value of a complex number. Since $I(\omega)$ decays at least as $O(1 / \omega)$ for large $\omega$, (see (7)), and since $\sin ^{2} \omega+\cos ^{2} \omega=1$, it follows from (40) that there exists a constant $K_{3}>0$ such that for $\omega$ sufficiently large, we have $D(\omega) \geq K_{3}$. Using this result and integration by parts in (34), we obtain the following estimate for large $\omega$ :

$$
\left(\omega c_{1}\right)^{2}=K_{1}\left(\int_{0}^{1} h^{2}(s) d s+\int_{0}^{1}\left(f^{\prime}\right)^{2}(s) d s+\|r\|^{2}+f^{2}(1)\right)
$$

for some $K_{4}>0$.

Since $\left\|(j \omega I-A)^{-1}\right\|$ decays at least as $O\left(\frac{1}{\omega}\right)$ for large $\omega$, by using (18), and (37)-(41), we obtain the following estimate for large $\omega$ :

$$
\int_{0}^{1} u_{x}^{2} d x+\int_{0}^{1} v^{2} d x+u^{2}(1)+w^{T} P w \leq K\left(\int_{0}^{1} h^{2}(s) d s+\int_{0}^{1}\left(f^{\prime}\right)^{2}(s) d s+\|r\|^{2}\right)
$$

for some $K>0$.

Since the imaginary axis belongs to the resolvent set $\rho(L)$ of the operator $L$, and since for each $\lambda \in \rho(L),(\lambda I-L)^{-1}$ is compact, it follows that for any $\Omega<\infty$,

$$
\sup _{\omega \leq \Omega}\left\|(j \omega I-A)^{-1}\right\|<\infty
$$

Hence, from (42)-(43), we conclude that the estimate given by (29) holds. Therefore, by the Theorem 2 , we conclude that the $C_{0}$-semigroup $T(l)$ generated by the operator $L$ is exponentially stable, that is (28) holds .

\section{Numerical Results}

In this section, to show the effect of the proposed controller given by (3)-(4) on the eigenvalues of the system given by (1)-(2), we present some numerical simulation results. By taking the Laplace transform, using (1)-(4), it can easily be shown that the eigenvalues of the system given by (1)-(4) are the roots of the following equation :

$$
\cosh s+g(s) \sinh s=0 \quad s \in \mathrm{C}
$$

where $g(s)$ is given by (7). When $g(s) \equiv 0$, (i.e uncontrolled system), the roots $\lambda_{n}$ of (44) are all on the imaginary axis and are given by $\lambda_{n}=j \omega_{n}, \omega_{n}=(2 n-1) \pi / 2, n=1,2, \ldots$.

To see the difference between the effects of the non-dynamic and dyamic boundary control on the eigenvalues, we first consider the non-dynamic boundary control given by (5) with the following parameter :

case $1: d=0.01$

The first five roots of (44), listed with increasing imaginary parts, are shown in the Table 1. As can be seen, the roots are uniformly bounded away from the imaginary axis, and the imaginary parts are close to the imaginary parts of the roots of the uncontrolled system given above. 
For some control applications it may be desirable to change the spectrum only over a prescribed frequency range. For example, the beam may be subject to a disturbance with a known frequency context. In this case, to reduce the effect of the disturbance, it may be desirable to introduce more damping only to the modes of the beam over the frequency range of the disturbance. Note that this could be achieved by increasing $d$, but in that case the remaining modes are also affected uniformly, and the required actuator energy will possibly increase, which may cause saturation in the controller.

To introduce more damping only to the lower modes, we propose the following controller transfer function :

$$
g(s)=d+\frac{K s}{s^{2}+2 \xi \omega_{0} s+\omega_{0}^{2}}
$$

where, $K, \xi$ and $\omega_{0}$ are positive constants.

The real part of $g(j \omega)$ is given by :

$$
\operatorname{Re}\{g(j \omega)\}=d+\frac{2 K \xi \omega_{0} \omega^{2}}{\left(\omega_{0}^{2}-\omega^{2}\right)^{2}+4 \xi^{2} \omega_{0}^{2} \omega^{2}}
$$

hence (6) is satisfied with $\gamma=d$. The maximum of $\operatorname{Re}\{g(j \omega)\}$ is obtained at $\omega=\omega_{0}$ and is given by :

$$
\max _{\omega \in \mathbb{R}} \operatorname{Re}\{g(j \omega)\}=d+\frac{K}{2 \xi \omega_{0}}
$$

and $\operatorname{Re}\{g(j \omega)\}$ decreases to $d$ as $\omega \rightarrow 0$ and as $\omega \rightarrow \infty$. Also note that the proposed dynamic controller does not increase uniformly the minimum of $\operatorname{Re}\{g(j \omega)\}$, that is :

$$
\inf _{\omega \in \mathbb{R}} \mathcal{R} e\{g(j \omega)\}=d
$$

Since we want to decrease $\operatorname{Re}\left\{\lambda_{1}\right\}$ and $\operatorname{Re}\left\{\lambda_{2}\right\}$, where $\lambda_{1}$ and $\lambda_{2}$ are first and second eigenvalues in the Table 1, respectively, from the reasoning above we conclude that a good choice for this purpose is $\omega_{0}=\operatorname{Im}\left\{\lambda_{1}\right\}$ or $\omega_{0 i}=\operatorname{Im}\left(\lambda_{2}\right\}$ We calculated the roots of $(44)$ for the following choices of the controller parameters :

case $2: d=0.01, K=1, \xi=0.05, \omega_{0}=\pi / 2$,

case $3: d=0.01, K=1, \xi=0.5, \omega_{0}=\pi / 2$,

case $4: d=0.01, K=1, \xi=0.5, \omega_{0}=3 \pi / 2$,

case $5: d=0.01, K=1, \xi=0.5, \omega_{0}=0.75 \pi$,

case $6: d=0, K=1, \xi=0.5, \omega_{0}=\pi / 2$,

In all cases, the first five roots of (44) arc given in the Tables 1-6. We note that these roots satisfy (44) wilh an error less than $10^{-8}$. As it can be seen from the Tables $2-5$, the resonant frequency $\omega_{0}$ of the controller transfer function determines the frequency (i.e. imaginary part) of the roots which are most affected. Table 6 also shows that in case the controller transfer function is strictly proper (i.e. $d=0$ ), the real parts of the roots are still negative, but not uniformly bounded away from the imaginary axis. The calculations show that in this case, the real parts of $100 t h, 200 t h$, and $500 t h$ roots are $-0.16 \times 10^{-4},-0.39 \times 10^{-5}$, and $-1.55 \times 10^{-6}$, respectively. 
$-0.01000033335334 \pm 1.57079632679490 i$

$-0.01000033335333 \pm 4.71238898038469 i$

$-0.01000033335333 \pm 7.85398163397448 i$

$-0.01000033335333 \pm 10.9955742875643 i$

$-0.01000033335333 \pm 14.1371669411541 i$

Table 1 : Eigenvalues for the case 1

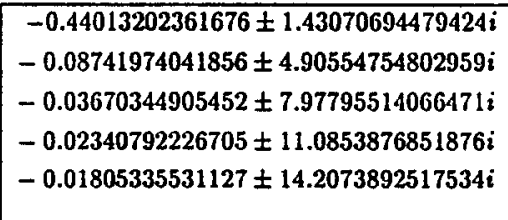

Table 3 : Eigenvalues for the case 3

$-0.18265022735691 \pm 1.35665728688789 i$

$-0.13399056866113 \pm 4.88840219600128 i$

$-0.05155450987594 \pm 7.97651348696870 i$

$-0.03049453791969 \pm 11.0851094770260 i$

$-0.02220453079721 \pm 14.2073060340342 i$

Table 5 : Eigenvalues for the case 5
$-0.06385789158546 \pm 0.98982624831444 i$

$-0.01989421933730 \pm 4.93382018349374 i$

$-0.01310205183168 \pm 7.98349414403971 i$

$-0.01152396109836 \pm 11.0873401366460 i$

$-0.01090777949229 \pm 14.2082946167328 i$

Table 2 : Eigenvalues for the case 2

$-0.03274928739695 \pm 1.50318874812767 i$

$-0.20798536990807 \pm 4.71120325463864 i$

$-0.10620974943388 \pm 7.95485691519529 i$

$-0.05554738410911 \pm 11.0811874857715 i$

$-0.03615909202903 \pm 14.2062183637846 i$

Table 4 : Eigenvalues for the case 4

$-0.43207714746874 \pm 1.43478150475045 i$

$-0.07669274060413 \pm 4.90608018631078 i$

$-0.02639771499343 \pm 7.97807016679770 i$

$-0.01324620331317 \pm 11.0854256884816 i$

$-0.00795407808717 \pm 14.2074044213073 i$

Table 6 : Eigenvalues for the case 6

\section{Conclusion}

In this paper, we considered a linear time-invariant distributed parameter system described by a onedimensional wave equation in a bounded domain (e.g. string, cable). We assumed that the system is clamped at one end and boundary control input is applied at the other end, (see (1)-(2)). To stabilize the system, we proposed a finite dimensional dynamic controller, (see (3)-(4)). The transfer function of the controller is restricted to be a strictly positive real function, (see the assumptions 1-3, ). We then prove that if the transfer function of the controller is strictly proper, then the resulting closedloop system is asymptotically stable; moreover following [6], one can show that the stability in this case can not be exponential, (see the Remark 1). We also show that if the controller transfer function is proper but not strictly proper, then the resulting closed-loop system is exponentially stable.

The class of stabilizing controllers proposed here is quite large and covers some previously proposed controllers as a special case (see (5)). This introduces extra degrees of freedom in designing controllers, which could be exploited in solving a variety of control problems, such as disturbance rejection, pole assignment, etc., while maintaining stability. Preliminary simulation studies show 
that by using the proposed controller, it could be possible to change the spectrum of the system given by (1)-(2) over a specified frequency range, while not disturbing the rest of the spectrum very much. To show this, we prersented some numerical simulation results in the section 4 . This point, and other applications of the proposed dynamic controller, require further investigation.

We also note that, the transfer function of the controller is allowed to be a strictly proper function. In this case, one can show that the open-loop map of the system is also strictly proper, (see the Remark 1). This is certainly a desirable property in proving certain stability robustness results, see [6]. However, the obtained stability is ouly asymptotical in this case, and not exponential. This shows that in constucting an algebraic framework for studying the stability of certain distributed parameter systems, to include the asymptotic stability in the stability definition, rather than exponential stability, might be a proper choice, see [1], [6]. However, this point also needs further investigation.

\section{References}

[1] F. M. Callier and J. Wilkin, "Distributed System Transfer Functions of Exponential Order," Int. J. Contr., vol. 43, No. 5, 1986, pp. 1353-1373.

[2] G. Chen,"Energy Decay Estimates and Exact Boundary Value Controllability for the Wave Equation in a Bounded Domain," J. Math. Pures. Appl., vol.58, pp.249-273,1979.

[3] G. Chen, M. C. Delfour, A. M . Krall and G. Payre, "Modelling, Stabilization and Control of Serially Connected Beams," SIAM J. Contr. Optimiz., vol. 25, pp. 526-546, 1987.

[4] G. Chen, S. G. Krantz, D. W. Ma, C. E. Wayne, and H. H. West, " The Euler-Bernoulli Beam Equation with Boundary Energy Dissipation," in Operator Methods for Optimal Control Problems, S. J. Lee, Ed., also in Lecture Notes in Pure and Applied Mathematics Series. New York : Marcell-Dekker, 1987, pp. 67-96.

[5] R. Datko, " Not All Feedback Stabilized Hyperbolic Systems are Robust with Respect to Small Time Delays in their Feedbacks," SIAM J. Contr. Optimiz., vol 26, No. 3, 1988, 697-713.

[6] A. J. Helmicki, C. A. Jacobson, and C. N. Nett, "Ill-Posed Distributed Parameter Systems: A Control Viewpoint," IEEE Trans. Auto. Contr., vol. 36, No. 9, 1991, pp. 1053-1057.

[7] F. L. Huang, "Characteristic Conditions for Exponential Stability of Linear Dynamical Systems in Hilbert Spaces," Annales Diff. Equations, vol 1, No. 1, 1985, pp. 43-53.

[8] T. Kato, Perturbation Theory for Linear Operators, 2nd. ed. New York : Springer Verlag, 1980.

[9] J. Lagnese, " Decay of Solutions of Wave Equations in a Bounded Domain with Boundary Dissipation," J. of Differential Equations, Vol. 50, pp. 163-182, 1983.

[10] J. Lagnese, Boundary Stabilization of Thin Plates, SIAM Studies in Applied Mathematics, vol. 10,1989

[11] L. Mejrovitch, Analitical Methods in Vibration, New York: MacMillan, 1967. 
[12] Ỏ. Morgül, "Orientation and Stabilization of a Flexible Beam Attached to a Rigid Body : Planar Motion," IEEE Trans. on Auto. Contr., vol 36, No. 8, pp. 953-963, 1991.

[13] Ö. Morgül, "Dynamic Boundary Control of an Euler-Bernoulli Beam," to appear in IEEE Trans. Auto. Contr., Apr. 1992.

[14] A. Pazy, Semigroups of Linear Operators and Applications to Partial Differential Equations, New York, Springer-Verlag, 1983.

[15] H. L. Royden Real Analysis, 2nd ed., New York : MacMillan, 1968.

[16] S. Saperstone, Semidynamical Systems in Infinite Dimensional Spaces, New York : Springer Verlag, 1981.

[17] S. M. Shahruz and Ö. Morgül, "Disturbance Rejection in Boundary Control Systems," Proceed. of 1989 ACC, Pittsburgh, PA, June 1989, pp. 1423-1424.

[18] M. Slemrod, "Stabilization of Boundary Control Systems," J. of Differential Equations, Vol. 22, pp. $402-415,1976$.

[19] J. J. E. Slotine, W. Pi, Applied Nonlinear Control, Englewood Cliffs, New Jersey: Prentice-Hall, 1991. 